\title{
Risk of peritoneal dialysis catheter-associated peritonitis following kidney transplant
}

\author{
Andrew M. Rizzi ${ }^{1}$ ｜ Stephen D. Riutta ${ }^{1}$ | Joshua M.Peterson ${ }^{1}$ | Galina Gagin ${ }^{1}$ | \\ Danielle M. Fritze $^{1}$ | Meredith Barrett ${ }^{1}$ | Randall S. Sung ${ }^{1}$ | Kenneth J. Woodside ${ }^{1}$ \\ Yee $\mathrm{Lu}^{2}$
}

\author{
${ }^{1}$ Section of Transplantation \\ Surgery, Department of Surgery, University of \\ Michigan, Ann Arbor, MI, USA \\ ${ }^{2}$ Division of Nephrology, Department of \\ Internal Medicine, University of Michigan, Ann \\ Arbor, MI, USA \\ Correspondence \\ Yee Lu, MD, MS, Assistant Professor of \\ Internal Medicine, Division of Nephrology, \\ Department of Internal Medicine, University \\ of Michigan Health System, Ann Arbor, MI, \\ USA. \\ Email: yeel@umich.edu \\ Funding information \\ University of Michigan Medical School \\ academic surgery interest group, SCRUBS
}

\begin{abstract}
Objective: Peritoneal dialysis (PD) patients have equivalent or slightly better kidney transplant outcomes when compared to hemodialysis (HD) patients. However, given the risk for postoperative infection, we sought to determine the risk factors for PD catheter-associated infections for patients who do not have the PD catheter removed at the time of engraftment.

Methods: Demographic and outcomes data were collected from 313 sequential PD patients who underwent kidney transplant from 2000 to 2015. Risk factors for postoperative peritonitis were analyzed using logistical regression.

Results: Of 329 patients with PD catheters at transplant, 16 PD catheters were removed at engraftment. Of the remaining 313 patients, $8.9 \%$ suffered post-transplant peritonitis. On univariate analysis, patients with peritonitis were significantly more likely to have used the PD catheter or HD within 6 weeks after transplant. Multivariate analysis had similar findings, with increased risk for those using the PD catheter after transplant, with a trend for those who underwent HD only within 6 weeks of transplant.
\end{abstract}

Conclusion: These results suggest that delayed graft function requiring any type of dialysis is associated with increased post-transplant peritonitis risk.

KEYWORDS

kidney transplantation, perioperative management, peritoneal dialysis catheter

\section{1 | INTRODUCTION}

Infections following transplantation are associated with reduced patient and allograft survival at 1 and 3 years after transplantation. ${ }^{1,2}$ The incidence of post-transplant peritonitis in peritoneal dialysis (PD) patients ranges from $8 \%$ to $43 \% .^{3-5}$ Given that PD patients have a $50 \%$ higher adjusted odds of receiving a kidney transplant when compared to hemodialysis (HD) patients, ${ }^{6}$ the management of PD catheters at the time of transplant could have a significant impact on patient and graft outcome. However, current guidelines are somewhat ambiguous regarding the management of the PD catheter post-transplant. ${ }^{7-10}$ Studies in pediatric patients suggested PD catheter use after transplantation was an effective treatment without infectious complications. ${ }^{10-12}$ European Best Practice Guidelines state "The catheter can be left in situ 3-4 months despite a functioning graft; nevertheless, earlier removal after successful transplantation is advisable." ${ }^{7}$ However, there is literature demonstrating increased incidence of infection, longer hospitalization, and increased risk for rejection associated with PD post-transplant. ${ }^{13}$ No prospective studies have been performed to address PD catheter management around the time of kidney transplantation. The purpose of this study was to compare outcomes of PD patients who 
TABLE 1 Demographics

\begin{tabular}{|cc|}
\hline General characteristics & $48(37-58)$ \\
\hline Age & $N=140(42.5 \%)$ \\
\hline Bemale sex & $28(24.78-32.33)$ \\
\hline BMI > 30 & $124(37.7 \%)$ \\
\hline African American & $51(16.3 \%)$ \\
\hline Caucasian & $242(77.3 \%)$ \\
\hline Dialysis vintage (months) & $62(26-137)$ \\
\hline Primary cause of ESRD & \\
\hline Hypertension & $49(14.9 \%)$ \\
\hline Diabetes & $93(28.3 \%)$ \\
\hline Polycystic kidney disease & $24(7.3 \%)$ \\
\hline Autoimmune & $47(14.3 \%)$ \\
\hline FSGS & $28(8.5 \%)$ \\
\hline Congenital & $15(4.6 \%)$ \\
\hline Other & $73(22.2 \%)$ \\
\hline Donor history & $73(22.2 \%)$ \\
\hline Living related & $79(24.0 \%)$ \\
\hline Living unrelated & $177(53.8 \%)$ \\
\hline Deceased & \\
\hline
\end{tabular}

Data listed as median (Q1-Q3) or number of cases (\%).

had PD catheters removed after their transplant surgery and identify risk factors for peritonitis.

\section{2 | METHODS}

A retrospective chart review was performed among 329 consecutive PD patients who had their primary kidney alone transplanted at the University of Michigan Transplantation Center between 2000 and 2015. End-stage renal disease patients greater than 18 years of age with PD as their dialysis modality at the time of transplantation between 2000 and 2015 were included in the study. Three hundred thirteen patients were included for analyses. Sixteen patients were excluded due to PD catheter removed at the time of engraftment based on surgeon preference and risk for delayed graft function. Patients were followed up for 6 weeks after transplantation for peritonitis and 365 days for patient mortality and graft loss/return to dialysis. There were six patient deaths with a functioning graft and no graft failures during the study period. Perioperative antibiotic prophylaxis, typically cefazolin, was utilized in accordance with center protocol. Post-transplant infectious prophylaxis included anti-Pneumocystis and anti-cytomegalovirus agents.

There were 136 recipients of living donor transplants and 177 recipients of deceased donor transplants. All patients had PD catheters managed by their primary nephrologist. Operative notes were reviewed to identify whether there was compromise of the peritoneal cavity during engraftment of the transplant. Ureteral stents were routinely used and removed by 6 weeks post-transplant.
Standard immunosuppression included an induction agent and maintenance with a calcineurin inhibitor, mycophenolate mofetil or mycophenolic acid, and prednisone. Induction with thymoglobulin was administered in patients for high degree of sensitization (PRA $>20 \%$, African American race, recipient of living unrelated transplant, patient undergoing desensitization or $\mathrm{ABO}$ incompatible transplant, patients $<65$ years old and receiving an extended criteria kidney, positive cross match due to donor-specific antibody or other anti-HLA antibody, presence of donor-specific antibody on any pretransplant sera regardless of cross match). Otherwise, patients were inducted with methylprednisolone without polyclonal or monoclonal antibody agents. In patients with delayed graft function, defined as requiring dialysis within the first week of transplant at our center, thymoglobulin at induction dosing was added post-transplant. Otherwise, there is no difference in the management of maintenance immunosuppression between patients with delayed graft function and immediate graft function.

The definition of peritonitis was based on that published by the International Society of Peritoneal Dialysis: peritonitis was identified based on peritoneal fluid white blood cell count greater than 100/ $\mu \mathrm{L}$ with at least $50 \%$ polymorphonuclear neutrophilic cells, a positive peritoneal fluid culture, or signs and symptoms of peritonitis based on clinical assessment. ${ }^{14}$ However, given that peritoneal signs are not reliably present on immunosuppressed patients, particularly those who have recently had induction immunosuppression, the absence of clinical signs or symptoms was not considered reliable for the purposes of this study. However, PD fluid samples were only collected if there was clinical concern for peritonitis or infection, fulfilling the last criterion for the diagnosis of peritonitis. Peritoneal fluid samples were collected under sterile conditions after a dwell of 2 hours by PD nurses when the physician has concern for peritonitis. Peritoneal fluid collection was obtained based on clinical concern for peritonitis.

\section{1 | Statistical analysis}

Demographic risk factors for peritonitis including age, race, body mass index (BMI), dialysis vintage (months), primary cause of ESRD, history of peritonitis, living vs deceased donor, induction therapy, documented peritoneum damage during operation, and dialysis modality at the time of transplant were obtained. Analyses were performed to determine risk factors for peritonitis in patients whose PD catheter remained in place postoperatively, compared to those who were removed intraoperatively.

Descriptive statistics were used to calculate percentages for dichotomous variables, and median with respective 25 th to 75 th percentiles for continuous variables. Categorical variables were compared using chi-square test. Continuous variables with normal distributions were compared using independent sample $t$ test, while the MannWhitney $U$ tests were used to compare continuous variables that had non-normal distributions. An initial univariate logistic regression analysis was performed, and all variables found to have a $P$-value of $\leq .20$ were included in a multivariate logistic model with stepwise selection, with a $P$-value of $\leq .10$ required to remain in the model. All comparisons 
were two-tailed, and a $P$-value of $\leq .05$ was considered significant throughout the analysis. Statistical analyses were performed using SAS version 9.3 (SAS Institute, Cary, NC, USA). The study protocol was approved by the institutional review board at the University of Michigan (IRB \#HUM00010092).

\section{3 | RESULTS}

Three hundred thirteen patients were included in the outcomes analysis. Demographic and clinical background data for the cohort of patients are shown in Table 1. The median age of the population was 48 years old (range: $37-58$ ), and $58 \%$ of the patients were male. Seventy-seven percent of the patients were Caucasian, and $16 \%$ were African American. The median BMI was 28 , and $38 \%$ of patients had BMI greater than 30 . The most common primary cause of ESRD was diabetes (28.3\% patients). The median duration of PD catheter use prior to transplantation was 630 days. For those who kept their catheter postoperatively, the median time to removal of the peritoneal catheter was 25 days after transplantation. Forty-nine (15.7\%) patients required dialysis within 6 weeks post-transplantation. There were six deaths during the first year post-transplant, but only one of these deaths was within 90 days. One patient death occurred with peritonitis listed as the cause of death.

There were 28 documented episodes of peritonitis in the 313 patients who did not have their peritoneal catheters removed at the time of transplant, 13 based on cell count and 15 episodes based on culture. Forty-six percent (13/28) were culture negative, 18\% (5/28) were due to coagulase-negative Staphylococcus, and $11 \%(3 / 28)$ were due to methicillin-resistant Staphylococcus aureus. The microbiologic organisms responsible for peritonitis are shown in Table 2.

Of the 49 patients requiring dialysis within the first 6 weeks posttransplant, 17 underwent HD using only a HD catheter or other vascular access, 16 utilized a dialysis catheter or other vascular access but had a PD catheter in place, and 33 utilized their existing PD catheter at some point for dialysis during of the first 6 weeks post-transplant, although they may have utilized HD for a portion of that time. There were no PD catheters placed for dialysis within the study period.

Risk factors associated with peritonitis in primary kidney transplant patients with PD catheters after transplantation were identified using univariate logistic regression. The results are presented in Table 3. PD catheter use within the first 6 weeks post-transplant (OR 3.46; 95\% Cl: 1.26, 9.4810-9.60; $P=.02$ ), HD catheter use within 6 weeks (OR 4.6, 95\% Cl: 1.34, 15.77, $P=.02$ ), HD or PD dialysis within the first 6 weeks post-transplant (OR $5.18 ; 95 \% \mathrm{Cl}$ : $1.71,15.74 ; P=.004)$ showed a statistically significant increased risk.

A pretransplant history of PD-associated peritonitis, HD within the first 6 weeks postoperatively, and PD use only within the first 6 weeks postoperatively were included in a multivariate analysis; "either HD or PD within 6 weeks" was excluded from the multivariate analysis, as it includes both other dialysis categories. The multivariate model had similar findings to the univariate analysis.
TABLE 2 Peritoneal fluid culture results

$\begin{array}{lc}\text { PD culture } & \text { Frequency } \\ \text { No growth } & 13 \\ \text { Coagulase (-) Staphylococcus } & 5 \\ \text { Methicillin-resistant Staphylococcus aureus } & 3 \\ \text { Pseudomonas aeruginosa } & 2 \\ \text { Vancomycin-resistant Enterococcus } & 1 \\ \text { Vancomycin-sensitive Enterococcus } & 1 \\ \text { Klebsiella species } & 1 \\ \text { Escherichia coli } & 1 \\ \text { Candida tropicalis } & 1\end{array}$

TABLE 3 Risk factors for peritonitis post-transplant

\begin{tabular}{|c|c|c|c|}
\hline Characteristic & Odds ratio & $95 \% \mathrm{Cl}$ & $P$-value \\
\hline $\begin{array}{l}\text { Age at transplant } \\
\text { (years) }\end{array}$ & 1.01 & $0.98,1.05$ & .40 \\
\hline Non-Caucasian race & 0.75 & $0.25,2.30$ & .61 \\
\hline Female & 1.06 & $0.45,2.48$ & .90 \\
\hline BMI & 1.02 & $0.98,1.06$ & .38 \\
\hline $\begin{array}{l}\text { Dialysis vintage } \\
\text { (months) }\end{array}$ & 1.004 & $0.99,1.02$ & .56 \\
\hline $\begin{array}{l}\text { DM as cause of } \\
\text { ESRD }\end{array}$ & 1.33 & $0.54,3.26$ & .53 \\
\hline $\begin{array}{l}\text { History of } \\
\text { PD-associated } \\
\text { peritonitis }^{a}\end{array}$ & 2.05 & $0.71,5.90$ & .19 \\
\hline $\begin{array}{l}\text { History of exit site } \\
\text { infection }\end{array}$ & $<0.001$ & $<0.001,>999$ & .9858 \\
\hline $\begin{array}{l}\text { Number of kidney } \\
\text { transplants }\end{array}$ & 1.94 & $0.90,4.15$ & .09 \\
\hline Deceased donor & 1.55 & $0.64,3.78$ & .34 \\
\hline Any induction agent & 1.37 & $0.49,3.85$ & .55 \\
\hline $\begin{array}{l}\text { Antithymocyte } \\
\text { antibody induction }\end{array}$ & 1.30 & $0.53,3.21$ & .57 \\
\hline $\begin{array}{l}\text { Renal biopsy within } \\
6 \mathrm{wk}\end{array}$ & 1.49 & $0.64,3.49$ & .36 \\
\hline $\begin{array}{l}\text { Either HD or PD } \\
\text { within } 6 \text { wk }\end{array}$ & 5.18 & $1.71,15.74$ & .004 \\
\hline $\begin{array}{l}\text { PD catheter use } \\
\text { within } 6 \mathrm{wk}^{\mathrm{a}}\end{array}$ & 3.46 & $1.26,9.48$ & .02 \\
\hline $\begin{array}{l}\text { HD catheter use } \\
\text { within } 6 w^{a}\end{array}$ & 4.60 & $1.34,15.77$ & .02 \\
\hline
\end{tabular}

${ }^{a}$ Variables included in the multivariable analysis.

PD catheter use within the first 6 weeks post-transplant showed a statistically significant rise in risk (OR 3.68; 95\% Cl: 1.21, 11.19, $P=.02)$. A trend toward significance was seen for patients who underwent HD within the first 6 weeks (OR 5.532; 95\% Cl: 0.94, 30.54; $P=.06$ ). A history of peritonitis was not a significant risk factor for developing peritonitis post-transplant (OR 2.24; $95 \% \mathrm{Cl}$ : $0.73,6.85 ; P=.16)$. 


\section{4 | DISCUSSION}

There was an $8.9 \%$ rate of peritonitis in this patient population, which is lower than what has been reported in some of the literature. ${ }^{13,15,16}$ Similar to the results from Warren et al, ${ }^{3}$ we found that patients requiring postoperative dialysis due to DGF had increased risk of peritonitis. In this study, either HD or PD within the first 6 weeks post-transplant was associated with a threefold to fivefold risk for peritonitis. Forty-six percent of the postoperative peritonitis episodes were culture negative and diagnosed based on cell count. Almost $30 \%$ of the peritonitis episodes were caused by staphylococcal infections. Peritoneal breach documented in operative reports was not associated with peritonitis. Staphylococcal infections have been associated with previous tunnel or exit site infections. ${ }^{4}$ These patients could also have altered flora due to the immunosuppression administered for their transplant or be nasal carriers of Staphylococcus.

Similar to Molnar et al, ${ }^{17}$ almost $16 \%$ of our patients required dialysis within the first 6 weeks post-transplantation. Sixty-seven percent of the patients requiring dialysis had HD, with almost 35\% dialyzing with either a HD catheter or other vascular access without a PD catheter present, and almost 33\% dialyzing with either a HD catheter or other vascular access with a PD catheter in place. The use of PD catheter at any time within the first 6 weeks post-transplantation was independently associated with an increased risk for peritonitis, when compared to those who did not use their PD catheters post-transplant.

Patients with slow graft function or DGF have more infections for multiple reasons, including prolonged hospitalization, dialysis requirement, and additional immunosuppressive therapy. A study by Gonwa et $\mathrm{al}^{18}$ outlined that patients with slow graft function or DGF had a longer length of stay and required more postoperative HD. GuimaraesSouza et al ${ }^{19}$ noted that patients with slow graft function or DGF had higher rates of infection than patients with immediate graft function. Our findings were consistent with the risk factors identified in this study.

This study has limitations. It is a single center study, with retrospective design. The center's PD catheter management protocol was to leave the catheters in place at the time of surgery until April of 2015. There may be differences in the patients before and after the change in the protocol that are not accounted for. There have been significant improvements in electronic storage of records, which may result in a difference in data fidelity for patients who were transplanted before and after the change in the data collecting mechanisms in the different electronic medical records. The retrospective design also limited our ability to determine the clinical indications for collection of peritoneal fluid for examination or to fully assess for false positives (eg, colonization). When combined with the unreliability of an abdominal examination for peritoneal signs in patients who have recently had induction immunosuppression, we chose to define peritonitis as having a WBC count of $>100$ cells/ $\mu \mathrm{L}$ in peritoneal fluid collected from patients with clinical suspicion for peritonitis. Furthermore, given the small number of patients with peritonitis, we might not be able to appreciate many of the risk factors for peritonitis in this single center study. With a larger, multicenter cohort, it is possible that further associations would emerge. The risk of peritonitis is impacted by the use of the PD catheter and possible peritoneal breach during kidney engraftment. While the former is available, the latter was not found to be reliably recorded in the operative record. Different practice patterns have existed over time, and among individual transplant nephrologists, on the willingness to use the PD catheter early after transplant, which could also impact the results. Finally, while this study was designed to evaluate risk factors for peritonitis associated with PD catheter presence post-transplant, HD catheters also pose a significant risk for infectious complications. As the utility of a PD catheter posttransplant is dependent not just on a risk-benefit analysis, but also on the local protocol or willingness to utilize the catheter, the applicability of this study will vary center to center.

In summary, post-transplant peritonitis diagnoses were rare. However, factors associated with dialysis requirement posttransplant placed patients with an indwelling PD catheter at an increased risk of peritonitis. Most patients requiring dialysis posttransplantation utilized HD as the primary modality in addition to PD, which placed patients at an increased risk for postoperative peritonitis. Given those practice patterns, PD catheter removal at the time of transplantation is a reasonable approach to care for these patients.

\section{ACKNOWLEDGEMENTS}

This study was supported by the University of Michigan Medical School academic surgery interest group, SCRUBS.

\section{CONFLICT OF INTEREST}

None of the authors have conflicts of interest to report.

\section{ORCID}

Kenneth J. Woodside (iD http://orcid.org/0000-0002-7495-3758

Yee Lu iD http://orcid.org/0000-0002-8002-6176

\section{REFERENCES}

1. Brayman KL, Stephanian E, Matas AJ, et al. Analysis of infectious complications occurring after solid-organ transplantation. Arch Surg. 1992;127:38-47; discussion 48

2. Witzke O, Schmidt C, Kohnle M, Lutkes P, Philipp T, Heemann U. Impact of febrile infections on the long-term function of kidney allografts. J Urol. 2001;166:2048-2052.

3. Warren J, Jones E, Sener A, et al. Should peritoneal dialysis catheters be removed at the time of kidney transplantation? Can Urol Assoc J. 2012;6:376-378.

4. Bakir N, Surachno S, Sluiter WJ, Struijk DG. Peritonitis in peritoneal dialysis patients after renal transplantation. Nephrol Dial Transplant. 1998;13:3178-3183.

5. Palmer JA, Kaiser BA, Polinsky MS, et al. Peritoneal dialysis catheter infections in children after renal transplantation: choosing the time of removal. Pediatr Nephrol. 1994;8:715-718. 
6. Mehrotra R, Chiu YW, Kalantar-Zadeh K, Bargman J, Vonesh E. Similar outcomes with hemodialysis and peritoneal dialysis in patients with end-stage renal disease. Arch Intern Med. 2011;171:110-118.

7. Dombros N, Dratwa M, Feriani M, et al. European best practice guidelines for peritoneal dialysis. 9 PD and transplantation. Nephrol Dial Transplant. 2005;20(Suppl 9):ix34-ix35.

8. Gokal R, Kost S. Peritoneal dialysis immediately post transplantation. Adv Perit Dial. 1999;15:112-115.

9. Melek E, Baskin E, Gulleroglu KS, Kirnap M, Moray G, Haberal M. Timing for removal of peritoneal dialysis catheters in pediatric renal transplant patients. Exp Clin Transplant. 2016;14(Suppl 3):74-77.

10. Arbeiter K, Pichler A, Muerwald G, et al. Timing of peritoneal dialysis catheter removal after pediatric renal transplantation. Perit Dial Int. 2001;21:467-470.

11. Vester U, Offner G, Hoyer PF, et al. End-stage renal failure in children younger than 6 years: renal transplantation is the therapy of choice. Eur J Pediatr. 1998;157:239-242.

12. Andreetta $B$, Verrina E, Sorino $P$, et al. Complications linked to chronic peritoneal dialysis in children after kidney transplantation: experience of the Italian Registry of Pediatric Chronic Peritoneal Dialysis. Perit Dial Int. 1996;16(Suppl 1):S570-S573.

13. Passalacqua JA, Wiland AM, Fink JC, Bartlett ST, Evans DA, Keay S. Increased incidence of postoperative infections associated with peritoneal dialysis in renal transplant recipients. Transplantation. 1999;68:535-540.

14. Li PK, Szeto CC, Piraino B, et al. ISPD Peritonitis Recommendations: 2016 Update on Prevention and Treatment. Perit Dial Int. 2016;36:481-508.
15. Maiorca R, Sandrini S, Cancarini GC, et al. Kidney transplantation in peritoneal dialysis patients. Perit Dial Int. 1994;14(Suppl 3):S162-S168.

16. O'Donoghue D, Manos J, Pearson R, et al. Continuous ambulatory peritoneal dialysis and renal transplantation: a ten-year experience in a single center. Perit Dial Int. 1992;12:242. 5-9.

17. Molnar MZ, Mehrotra R, Duong $U$, et al. Dialysis modality and outcomes in kidney transplant recipients. Clin J Am Soc Nephrol. 2012;7:332-341.

18. Gonwa TA, Mai ML, Smith LB, Levy MF, Goldstein RM, Klintmalm GB. Immunosuppression for delayed or slow graft function in primary cadaveric renal transplantation: use of low dose tacrolimus therapy with post-operative administration of anti-CD25 monoclonal antibody. Clin Transplant. 2002;16:144-149.

19. Guimaraes-Souza NK, Dalboni MA, Camara NC, Medina-Pestana JO, Paheco-Silva A, Cendoroglo M. Infectious complications after deceased kidney donor transplantation. Transplant Proc. 2010;42:1137-1141.

How to cite this article: Rizzi AM, Riutta SD, Peterson JM, et al. Risk of peritoneal dialysis catheter-associated peritonitis following kidney transplant. Clin Transplant. 2018;32:e13189. https://doi.org/10.1111/ctr.13189 